Research Paper

\title{
CSN6 promotes the cell migration of breast cancer cells by positively regulating Snaill stability
}

\author{
Jie Mou'1,2,3\#, Lulu Wei1,4\#, Jia Liang1, Wenqi Du5, Dongsheng Pei ${ }^{\circledR}$ \\ 1. Department of Pathology, Xuzhou Medical University, Xuzhou, China \\ 2. Jiangsu Key Laboratory of New drug and Clinical Pharmacy, Xuzhou Medical University, Xuzhou, China. \\ 3. School of Pharmacy, Xuzhou Medical University, Xuzhou, China. \\ 4. Center for Translational Medicine and Jiangsu Key Laboratory of Molecular Medicine, Medical School of Nanjing University, Nanjing, China. \\ 5. Department of Human Anatomy, Xuzhou Medical University, Xuzhou, China. \\ \#These authors contributed equally to this work. \\ $\triangle$ Corresponding author: Dongsheng Pei, Department of Pathology, Xuzhou Medical University, Xuzhou, China. Tel: +8613952269686. E-mail: dspei@xzhmu.edu.cn.
}

(C) The author(s). This is an open access article distributed under the terms of the Creative Commons Attribution License (https://creativecommons.org/licenses/by/4.0/). See http://ivyspring.com/terms for full terms and conditions.

Received: 2020.07.02; Accepted: 2020.09.09; Published: 2020.10.01

\begin{abstract}
Background: CSN6, a subunit of the highly conserved constitutive photomorphogenesis 9 (COP9) signalosome (CSN), has been reported to be implicated in tumor progression in various kinds of malignant tumors. However, the mechanism underlying CSN6 in the tumor development of breast cancer has not yet been fully elucidated.

Methods: CSN6 staining in breast cancer tissues and paracancerous tissues was measured by tissue microarray (TMA) technology. The metastatic effect of CSN6 was measured by cell migration assay. Co-immunoprecipitation study was used to show the interaction between the protein CSN6 and Snaill. Ubiquitination assay was performed to validate whether ubiquitination is involved in the upregulation of Snail 1 by CSN6. The impact of CSN6 on tumor metastasis in vivo was analyzed using xenotransplantation experiments in $\mathrm{BALB} / \mathrm{c}$ mice.

Results: Here, we demonstrated that CSN6 expression was dramatically increased in breast cancer tissues compared with paired adjacent cancerous tissues. CSN6 promoted the cell migration and wound healing abilities in breast cancer cell lines. Also we showed that CSN6 associates with Snaill and enhances Snaill protein level by inhibiting the ubiquitin-mediated degradation of Snaill. Thus, CSN6 is involved in positively regulating the stability of Snaill. We further proved that CSN6 protein level was positively correlated with the Snaill expression in xenograft model.

Conclusion: These findings provide new insight into applicability of using the CSN6-Snaill axis as a potential therapeutic target in breast cancer.
\end{abstract}

Key words: CSN6, Snail1, cell migration, breast cancer

\section{Introduction}

Breast cancer remains a public health issue on a global level and its survival rate falls from $90 \%$ for localized to $20 \%$ for metastatic disease [1, 2]. Nowadays, the identification of effective interventions to avoid breast cancer is still challenging [3]. Stromal invasion and metastasis to distant organs or regional lymph nodes are the characteristics of fully developed breast cancer [4]. In addition, obvious metastasis frequently indicates an incurable and chronic disease. The amount of biomolecular markers and targeted therapeutic drugs for breast cancer management has rapidly increased in the past decades. Therefore, further identifying reliable biomarkers and exploring novel targets, is urgently needed for the long-term survival of patients with breast cancer.

The highly conserved constitutive photomorphogenesis 9 (COP9) signalosome (CSN) is an evolutionarily conserved multisubunit protein complex that resembles the 195 lid of the $26 \mathrm{~S}$ proteasome that is common to all eukaryotes and found in plants and animals [5-7]. The COP9 signalosome has been postulated to be associated with protein degradation because of its sequence 
homology with the 'lid' complex of the $26 \mathrm{~S}$ proteasome in mammals [8]. CSN6, a critical subunit of COP9 signalosome, is involved in ubiquitin-mediated degradation of important proteins implicated in cell cycle progression and signal transduction that relies on the Mprl/Pad1 N-terminal domain [9-13]. Furthermore, CSN6 can also coordinate with E3 ligase to mediate ubiquitination and degradation of cancer associated proteins [14]. Previous studies have identified that CSN6 was implicated in tumor progression, because it is overexpressed in multiple common types of cancers, such as cervical cancer, thyroid cancer, colorectal cancer, breast cancer, lung cancer, hepatocellular carcinoma and glioblastoma [15-19]. In particular, the expression profiles of CSN6 in human cancer patient database from Gene Expression Omnibus and Oncomine illustrate that CSN6 is overexpressed in $>50 \%$ of cases of glioblastoma, pancreatic cancer and breast cancer [20]. However, the molecular mechanism for CSN6 in breast cancer remains obscure.

The epithelial-mesenchymal transition (EMT) is a highly conserved biological process that shifts epithelial cells to a mesenchymal phenotype initially in embryonic development [21]. It is well accepted that EMT is critical for the progression of tumor occurrence, migration and metastasis [2]. Snail1, a key transcription factor of EMT, was identified in Drosophila, controls large-scale cell movement during neural crest delamination and mesoderm formation [22]. Snail1 overexpression was detected in both epithelial and endothelial cells of invasive cancer [23]. In addition, Snail1 is related with tumor progression and poor outcome in patients in human malignancies $[21,24]$. In breast cancer, Snail1 correlates with the tumour grade, poor clinical parameters, a high rate of recurrence, distant metastasis and nodal metastasis for invasive ductal carcinoma [25-28]. Furthermore, Snail1 also brings resistance to apoptosis and produces breast cancer stem cell (CSC)like characteristics by blocking Snail1 ubiquitination [29-31]. These suggest that Snail1 is a possible target for therapeutic intervention. However, a comprehensive account of the mechanisms of Snail1 stabilization in breast cancer remains unclear.

In the current study, we utilized unbiased approaches to characterize the mechanism of CSN6 overexpression during the breast cancer tumorigenesis. Furthermore, we identified that CSN6 enhancement leads to Snail1 stabilization and promotes metastasis of breast cancer cells through inhibiting Snail1 ubiquitination. Our findings provided a thoughtful understanding of novel CSN6-Snail1 signaling in promoting breast cancer metastasis.

\section{Materials and Methods}

\section{Patients and samples}

The tissue microarray (TMA) was consisted of 52 cases breast cancer tissues and paired paracancerous tissues, which were collected from the Affiliated Hospital of Xuzhou Medical University. All the patients underwent mastectomy and postoperative adjuvant therapy at Affiliated Hospital of Xuzhou Medical University from March 2012 to May 2016. Detailed clinical information of specimen was recorded accurately, such as clinicopathological parameters including age, TNM stage, lymph node metastasis and others like birth place, marriage and surgery date.

\section{Ethics statement}

Patient tissue samples were obtained with informed consent, under the protocol approved by the ethical review board of the Affiliated Hospital of Xuzhou Medical University. Animal experiments were performed in strict accordance with the protocols approved by the Institutional Animal Care and Use Committee of Xuzhou Medical University.

\section{Immunohistochemistry and evaluation}

After being baked at $65{ }^{\circ} \mathrm{C}$ for $2 \mathrm{~h}$, TMA slides were dewaxed with dimethylbenzene and then rehydrated with graded alcohol and distilled water. For retrieving antigen, TMA slides were performed in a microwave oven heated at $95^{\circ} \mathrm{C}$ with $10 \mathrm{mM}$ citrate buffer ( $\mathrm{pH}$ 6.0). Endogenous peroxidases were quenched by $3 \% \mathrm{H} 2 \mathrm{O} 2$ for $20 \mathrm{~min}$. The slides were treated with normal goat serum to inhibit nonspecific staining, then incubated with anti-CSN6 (1:50 dilution, Santa Cruz Biotechnology, USA) overnight at $4{ }^{\circ} \mathrm{C}$ and then were incubated with a secondary antibody for $1 \mathrm{~h}$. Diaminobenzidine (DAB; Zhongshan biotech, Beijing, China) was used to produce a brown precipitate. After hematoxylin counterstain and dehydration were completed, the slides were sealed. The immunoreactivity was assessed blindly by two independent observers by microscopy (ZEISS AXIO Scope. A1). The expression of CSN6 was graded as positive when $\geq 5 \%$ of tumor cells showed immunopositivity. Biopsies with $<5 \%$ tumor cells showing immunostaining were considered negative.

\section{Cell lines and cell culture}

Human normal breast epithelial cell line MCF10A and human breast cancer cell lines BT549, T47D and MDA-MB-231 were obtained from the Shanghai Institute of Biochemistry and Cell Biology, 
Chinese Academy of Science (Shanghai China). MCF10A cells were cultured in DMEM/F12 Medium, BT549 were cultured in RPMI-1640 Medium, T47D were cultured in DMEM Medium while MDA-MB231 were cultured in L15 Medium supplemented with $10 \%$ fetal bovine serum, $100 \mathrm{U} / \mathrm{ml}$ penicillin, 100 $\mu \mathrm{g} / \mathrm{ml}$ streptomycin, and incubated in a $37{ }^{\circ} \mathrm{C}$ humidified incubator with $5 \% \mathrm{CO}_{2}$.

\section{Stable cell line generation}

The stable CSN6 overexpressed MDA-MB-231 cells were established by infected with lentiviruses, in which CSN6-control expression vectors and CSN6overexpression vectors were respectively packed by Gene-Pharma (Soochow, China). MDA-MB-231 cells were infected with lentivirus for $48 \mathrm{~h}$, and then were selected with $2 \mathrm{ng} / \mathrm{ml}$ puromycin for 2 weeks, with the medium refreshed every 3 days.

\section{Small interfering RNA and transient transfections}

Small interfering RNA (siRNA) specific for CSN6 (siCSN6) and siRNA NC were purchased from Gene-Pharma (Soochow, China) and transfected by siLentFect Lipid Reagent (Bio-Rad, Hercules, CA, USA) according to the manufacturer's protocol when breast cancer cells were grown to $50 \% \sim 60 \%$ confluency. Six hours after transfection, the medium containing transfection reagents were removed and incubated in fresh medium. The siRNAs sequences were described as follows: si-CSN6 sense: $5^{\prime}$-CCGUG GAAGAGAAGAUUAUTT-3', siCtrl sense: 5'-UUCU CCGAACGUGUCACGUTT-3'.

\section{Western blot analysis}

After digestion and centrifugation of the cells, protein concentration was determined by bicinchoninic acid kit (BCA, Beyotime Biotechnology, Beijing, China). $100 \mu \mathrm{g}$ proteins were applied onto $12.5 \%$ SDS polyacrylamide gel (SDS-PAGE) for electrophoresis and then transferred onto nitrocellulose membrane, and probed with the primary antibodies (CSN6, 1: 2000, Enzo Life Sciences; Snail1, 1: 1500, Abcam; $\beta$-actin, 1: 2000, Zhong-shan biotech). After incubating overnight at $4{ }^{\circ} \mathrm{C}$ with the primary antibody, membranes were washed with TBS/0.05\% Tween-20 (TBST) and incubated with a secondary antibody (1:10000, VICMED) for 2 hours at room temperature. The membranes were then washed and scanned on the chemiluminescence imaging analysis system (Tanon, Biotechnology, Shanghai, China).

\section{Reverse transcription-polymerase chain reaction (RT-PCR)}

Total RNA was extracted with Trizol Reagent (Thermo Fisher). The initial reaction uses the reverse transcriptase enzyme to generate DNA from RNA. Firstly the reaction was set up on ice in a $0.2 \mathrm{ml}$ thin-walled PCR tube. Incubate samples in a thermocycler for $1 \mathrm{~h}$ at $37-42{ }^{\circ} \mathrm{C}$. And the reversetranscribed single-stranded DNA was denatured at 95 ${ }^{\circ} \mathrm{C}$ for $2 \mathrm{~min}$ and place on ice. Then pour a $1 \%$ agarose gel with ethidium bromide in 1×TAE. Run $5-10 \mathrm{ml}$ of the PCR reaction along with a DNA ladder at $100 \mathrm{~V}$ for 30-45 min. Finally the gel was visualized using a UV gel documentation system.

\section{Co-immunoprecipitation}

Cell lysates for immunoprecipitation were incubated on a rocker with indicated antibody (CSN6, 1: 50, Enzo Life Sciences) at $4{ }^{\circ} \mathrm{C}$ overnight for coimmunoprecipitation (co-IP) assay. The lysis buffer contained a cocktail of protease/phosphatase inhibitors (sigma) and cell lysates were immunoprecipitated by Protein A/G beads (Santa Cruz). Then the beads were centrifuged at a low speed for $10 \mathrm{~min}$ and supernatant was discarded. Dried beads were mixed with $1 \times$ loading buffer and boiled for $5 \mathrm{~min}$. Lysate samples were loaded onto gels following western blot analysis.

\section{Migration assay}

Transwell chambers with a pore size of $8 \mu \mathrm{m}$ were applied to examine cell migration. $1 \times 10^{4}$ breast cancer cells were seeded in serum-free medium in the upper chamber. The medium containing $10 \%$ FBS was added to the lower chamber. After cultured for $24 \mathrm{~h}$ in a $37^{\circ} \mathrm{C}$ incubator, the cells were fixed in methanol and stained with leucocrystal violet. Cells in upper chamber were removed and the number of cells which traversed the membrane was determined by counting the leucocrystal violet stained cells. Stained cells were counted under a microscope in the whole field.

\section{Wound healing assay}

The transfected BT-549 and MDA-MB-231 cells were seeded in 6-well plates. When cell culture reached about $95 \%$ confluence, the cell monolayer was slowly scratched with a sterile $200 \mu$ pipette tip. We washed the well with fresh medium to remove cellular debris and cultured cells again. The wound was imaged at 0 and $24 \mathrm{~h}$. All assays were repeated three times.

\section{Ubiquitination assay}

BT-549 and MDA-MB-231 cells were transfected with indicated plasmids. After 24 hours, cells were treated with $50 \mu \mathrm{g} / \mathrm{mL}$ of MG132 (Selleck Biotechnology, USA) for $6 \mathrm{~h}$. The ubiquitinated proteins were immunoprecipitated with anti-Snail1. The protein complexes were then resolved by SDS- 
polyacrylamide gel and probed with anti-ubiquitin to visualize the level of ubiquitination.

\section{Xenograft mouse metastatic model}

Twenty female 6-week-old BALB/c nude mice were purchased from Beijing Huafukang Bioscience (Beijing, China). Stable MDA-MB-231 cells (LV$\mathrm{CSN6}^{\mathrm{OE}}$ and LV-NC) were concentrated to $2 \times 10^{6} / 100$ $\mu 1$ PBS and injected into mice via the lateral tail vein. After 30 days, all mice were euthanized and lungs were then excised and photographed. The number of metastatic tumors per lung were counted and recorded. Then, the lung tissues were harvested, embedded, fixed, and prepared for H\&E, IHC staining and western blot.

\section{Statistical analysis}

Data were analyzed using SPSS 16.0 software. Statistical significance of Student's $t$-test was presented for two-group comparisons. More than two groups were examined by one-way or two-way analysis of variance. The correlations between CSN6 expression and clinicopathologic characteristics were analyzed using Pearson's chi-square test or Fisher's exact test in breast cancer tissues and para-cancer tissues. Differences between groups were statistically significant when $P<0.05$.

\section{Results}

\section{CSN6 is increased in breast cancer tissues and is associated with clinicopathologic parameters in breast cancer}

To investigate whether the expression of CSN6 is changed in breast cancer cells, western blot analysis was performed to examine the CSN6 expression in normal breast epithelial cell line MCF10A and three human breast cancer cell lines. Results showed that the expression of CSN6 in breast cancer cells is higher than that in normal breast epithelial cells (Figure 1A). To evaluate the correlation between CSN6 expression and clinicopathological parameters (according to TNM classification), we collected breast cancer tissues and corresponding adjacent tissues from 52 breast cancer patients. Then CSN6 expression was analyzed using IHC in the TMA of 52 breast cancer specimens to investigate the clinical implication of CSN6 protein. We found that CSN6 expression is localized in the cytoplasm. In addition, the expression of CSN6 was higher in cancerous tissues than in paracancerous tissues (Figure 1B). In paracancerous tissues, positive CSN6 staining was recorded in $23.1 \%$ (12 of 52 cases). Of the 52 patients with breast cancer tissues, positive expression of CSN6 was observed in 59.6\% (31 of 52 cases) (Table 1). Because TNM stage is an important prognostic marker for breast cancer patients, so we investigated if CSN6 expression correlates with TNM stage. Our data demonstrated that CSN6 showed a significant correlation with histological grade $(P<$ 0.05 , Table 1). Meanwhile, statistical analysis revealed that increased CSN6 expression significantly correlated with depth of invasion pT $(P<0.05$, Table 1$)$ and lymph node metastasis $\mathrm{pN}(P<0.05$, Table 1$)$.

Table 1. Clinical characteristics of 52 breast cancer patients and CSN6 expression

\begin{tabular}{lllll}
\hline Pathology Character & $\mathrm{N}$ & \multicolumn{2}{l}{ CSN6 staining } & P Value \\
\cline { 3 - 4 } & & Negative & Positive & \\
\hline Tissue & & & & $0.0001^{* * *}$ \\
Cancerous & 52 & 21 & 31 & \\
$\begin{array}{l}\text { Paracancerous } \\
\text { Age (year) }\end{array}$ & 52 & 40 & 12 & 0.508 \\
$\leq 55$ & & & & \\
$>55$ & 27 & 15 & 12 & $0.016^{*}$ \\
TNM Stage & 25 & 13 & 12 & \\
I & & & & \\
II & 9 & 8 & 1 & $0.024^{*}$ \\
III & 30 & 12 & 18 & \\
pT status & 13 & 4 & 9 & \\
pT1 & & & & \\
pT2 & 11 & 9 & 2 & \\
pT3 & 29 & 10 & 19 & \\
pT4 & 7 & 3 & 4 & \\
pN status & 5 & 0 & 5 & \\
pN0 & & & & \\
pN+ & 34 & 22 & 12 & \\
\hline
\end{tabular}

\section{CSN6 promotes breast cancer cells migration in vitro}

It is acknowledged that malignant cells are characterized by possessing the capabilities of adhesion and migration. Hence, we investigated the role of CSN6 in motility of breast cancer cells. To access the effect of CSN6 on cell migration ability, western blot was performed and showed that siCSN6 and CSN6 overexpression plasmid were successfully transfected into breast cancer cells (Figure 2A). Cell migration was measured by transwell assays, and we found that CSN6 knockdown BT549 and MDA-MB-231 cells could harbor weaker abilities to penetrate through the inserts (Figure 2B). On the contrary, overexpression of CSN6 increased the number of cells passing through the inserts (Figure 2C). Wound healing assays showed that knockdown of CSN6 decreased the capacity for BT549 and MDA-MB-231 cell motility (Figure 2D). Furthermore, a significant increase was revealed in the wound closure of CSN6 overexpression cells when compared with the control cells (Figure 2E). Overall, the results indicated that CSN6 promoted the migration in breast cells in vitro. 

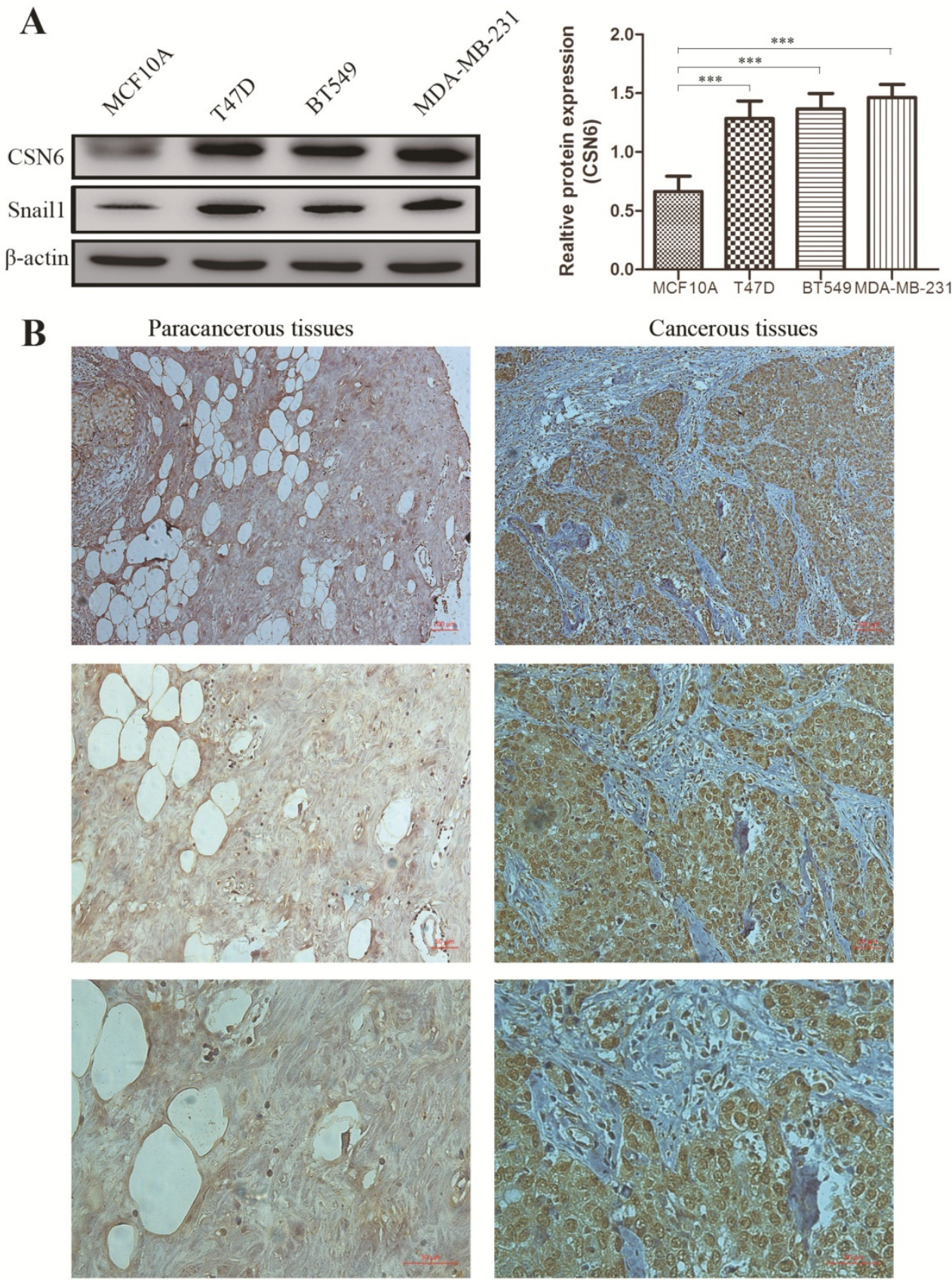

Figure 1. CSN6 expression is increased in human breast cancer cells and tissues. (A) Western blot analysis was used to evaluate the CSN6 and Snaill expression in various breast cancer cell lines and normal breast epithelial cell line MCF10A. The data represent means \pm S.D. $* * * P<0.001$. (B) Representative photos of CSN6 expression patterns in breast cancer tissues. The right side shows the breast cancerous tissues, whereas the left side depicts matched paracancerous tissues (original magnification, 100x). The magnifying detail of the immunohistochemical analysis for each case can be shown on the middle panel (original magnification, 200x) and bottom panel (original magnification, $400 \times)$.

\section{CSN6 stabilizes Snail1 by impeding Snail1 ubiquitination}

It is identified that Snail1 is the main regulator of EMT and EMT is the process of cancer cells invasion and migration. In addition, Snail1 is a zinc finger transcription factor, and plays an important role in tumorigenesis through silencing the expression of E-cadherin and inducing EMT. To investigate the mechanism by which CSN6 regulates tumor metastasis in breast cancer cells, Western Blot was performed to examine the relationship between CSN6 and Snail1 protein levels in BT549 and MDA-MB-231 cells. In Figure 3A, data showed that the protein level of Snail1 decreased after CSN6 knockdown and the expression level of Snail1 protein increased after CSN6 overexpression in the breast cancer cells. Meanwhile, the expression of Snail1 in breast cancer cells is higher than that in normal breast epithelial cells which is consistent with CSN6 expression (Figure 1A). Furthermore, CSN6 could positively regulate the stability of Snail1 in a dose-dependent 
manner (Figure 3B). The above results prompted us to study how CSN6 up-regulates Snail1 protein. We found that the mRNA level of Snail1 did not change after CSN6 overexpression in breast cancer cell lines (Figure 3C). This suggested that Snaill expression may be regulated by CSN6 at or after translation level. The results of co-immunoprecipitation showed that CSN6 and Snail1 proteins bound to each other (Figure 3D). We next investigated how CSN6 interacted with Snail1 to regulate the protein level of Snail1. Breast cancer cells were transfected with the indicated plasmids and treated with the proteasomal inhibitor MG132. Results showed that Snail1 protein levels increased in the presence of both CSN6 and MG132 (Figure 3E). Then we performed ubiquitination assay and found that CSN6 overexpression decreased the ubiquitination level of Snail1 (Figure 3F). Taken together, these results indicated that CSN6 promotes Snail1 stability through reducing ubiquitination.

\section{CSN6 promotes tumor metastasis via up- regulating snail 1 in xenograft model}

To identify the role of CSN6 in the metastatic potency of breast cancer cells in vivo, lentiviruses packed with CSN6-NC or CSN6 overexpression vectors were transfected into MDA-MB-231 cell lines, and the fluorescence intensity was detected by Olympus light microscope (Figure 4A). CSN6 overexpression cells were injected into tail veins of the nude mice to establish a tumor metastasis model and compared numbers of lung nodules with those among control nude mice injected with CSN6-NC cells. After 30 days, the nude mice were sacrificed and the lung tissue was dissected for observation. We found that the number of metastatic lung nodules in CSN6-overexpression mice were significantly higher than the control group (Figure 4B). To detect the CSN6 expression and snail1 protein levels in vivo, western blot analysis was performed to examine the extracted protein of metastasis tumor. The results indicated that the CSN6 and snail1 expression levels were up-regulated in CSN6 overexpression group versus control group (Figure 4C). Furthermore, the lung tissues were paraffin embedded for subsequent analysis and the expression of CSN6, Snail1 was detected via Immunohistochemistry staining (Figure 4D). The results demonstrated that CSN6 promoted tumor metastasis in vivo and up-regulated the protein level of Snail1, which was consistent with the results mentioned in vitro experiments.

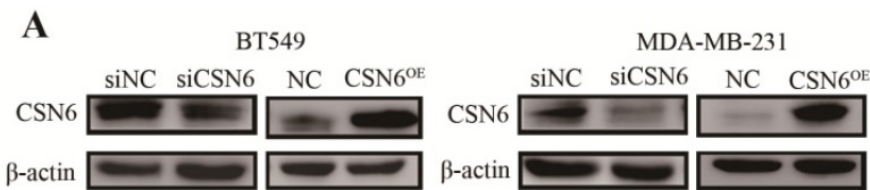

B
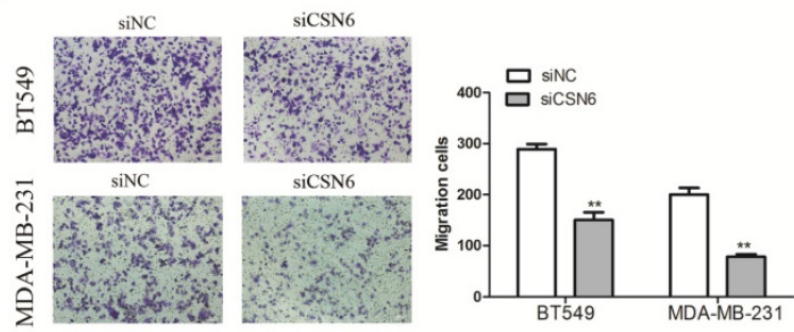

C
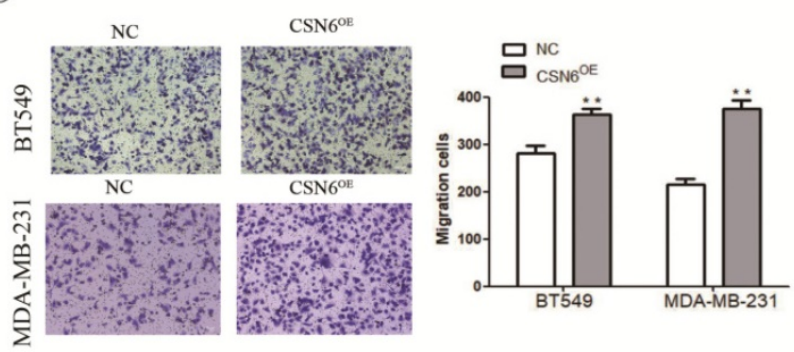

D
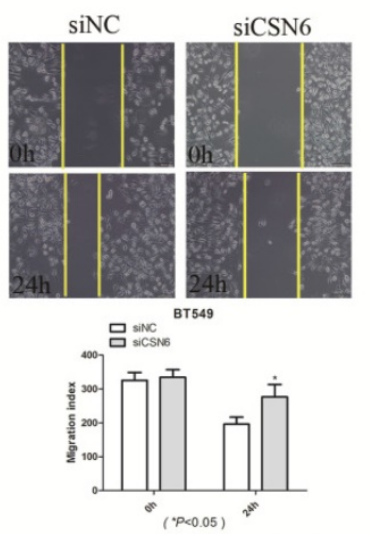

$\mathbf{E}$

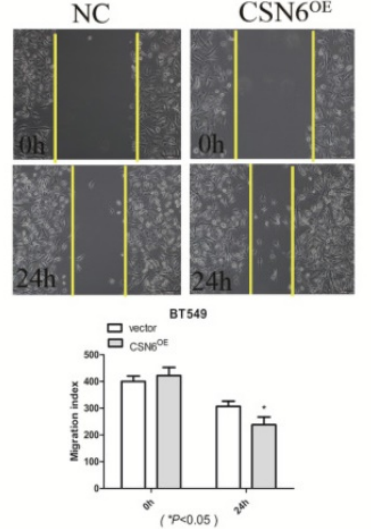

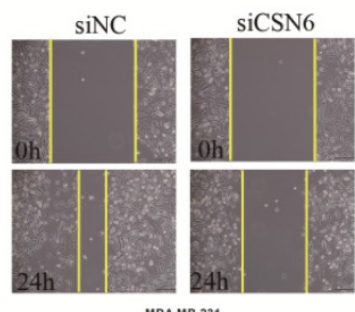
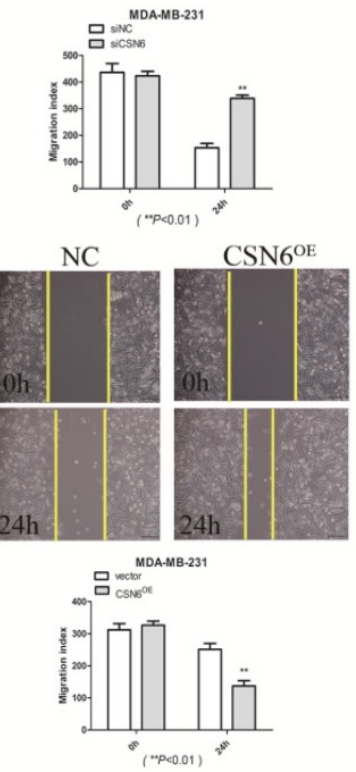

Figure 2. CSN6 promotes migration and invasion in breast cancer cells. (A) Western blot was used to detect the expression efficiency of CSN6 when CSN6 gene vector or Si-CSN6 was transfected into two kinds of breast cancer cells. (B-C) Cell migration assays were performed in BT-549 and MDA-MB-231 cells after CSN6 knockdown or expression, respectively. (D-E) The breast cancer cells motilities were measured through testing the wound closure after CSN6 overexpression and knockdown in BT-549 and MDA-MB-231 cells, respectively. The data represent means \pm S.D. $* P<0.05$, $* * P<0.01$, $* * * P<0.001$. 
A

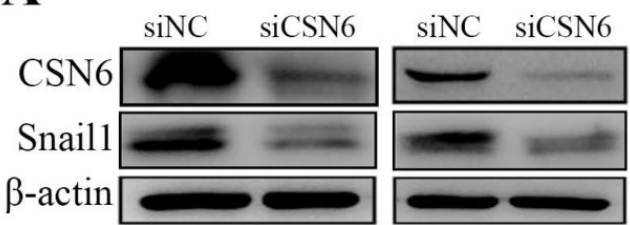

B

C

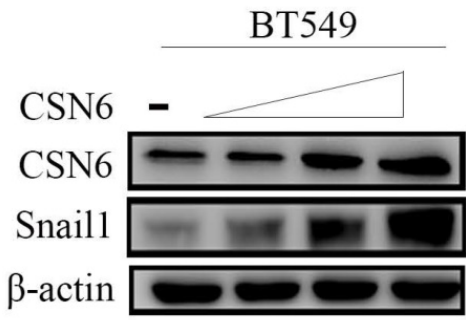

BT549

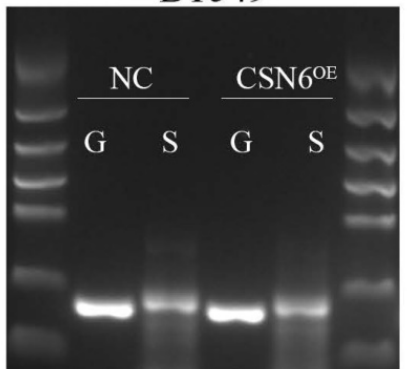

BT549

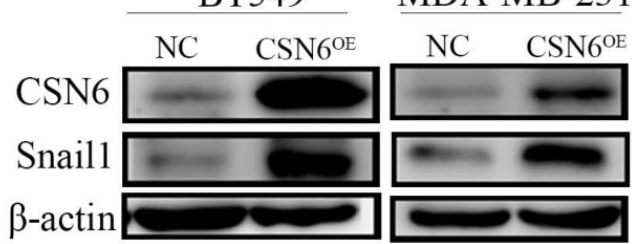

MDA-MB-231

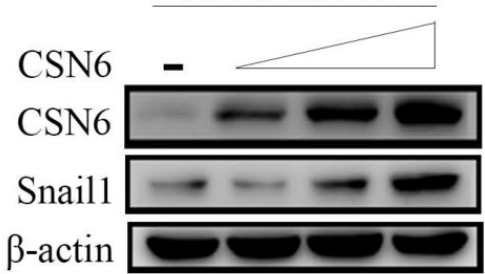

MDA-MB-231

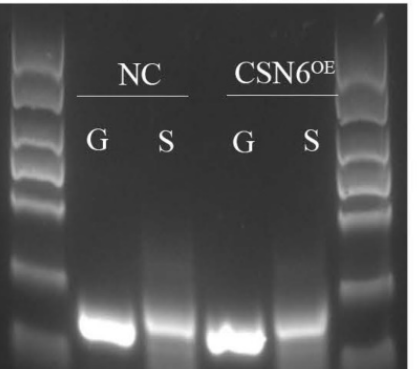

D

(G: GAPDH S: Snaill)

IB:Snaill
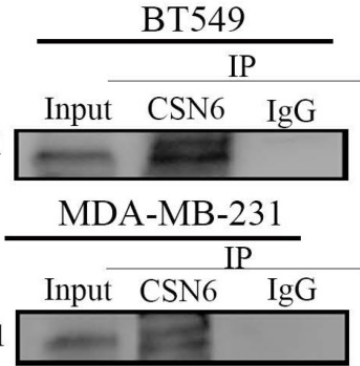

$\mathbf{F}$

IB:Snaill

$\mathbf{E}$
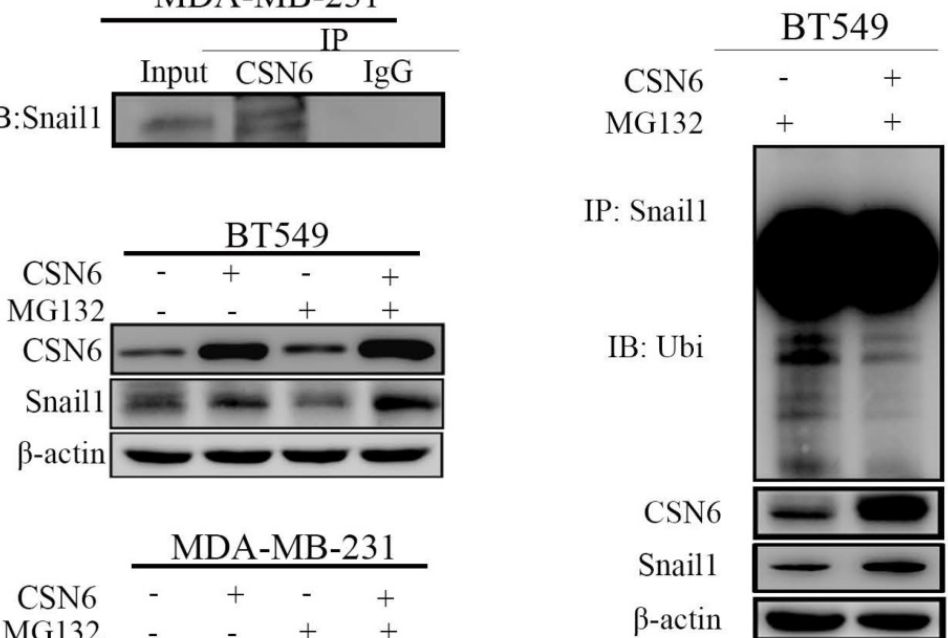

MDA-MB-231

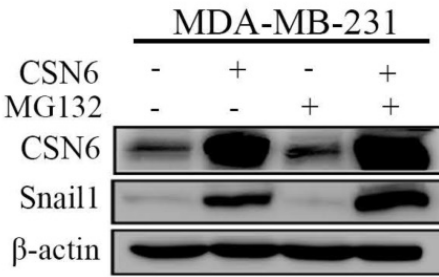

$\beta$-actin

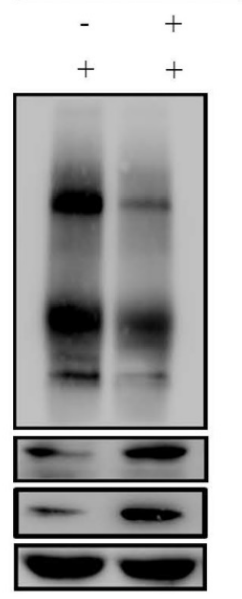

Figure 3. CSN6 positively regulates the expression of Snail1. (A) Western blot analysis of Snaill expression after CSN6 overexpression or knockdown in BT-549 and MDA-MB-231 cells. (B) CSN6 enhanced the protein level of Snail1 in a dose-dependent ( $2 \mu \mathrm{g}, 4 \mu \mathrm{g}, 6 \mu \mathrm{g})$ manner in breast cancer cell lines. (C) The mRNA levels of Snaill were checked when BT-549 and MDA-MB-231 cells were transfected with plasmids expressing CSN6 or pcDNA3.1. (D) The breast cancer cells lysates were analyzed by immunoprecipitation with CSN6 and were immunoblotted with anti-Snaill. CSN6 interacted with endogenous Snaill. (E) CSN6 prevents Snaill degradation via proteasomal degradation. BT-549 and MDA-MB-231 cells were transfected with CSN6-expression vectors and treated with or without MG132 for 6 h, then subjected to Western blot analysis using an anti-Snaill or anti-actin antibody. (F) BT-549 and MDA-MB-231 cells were transfected with control vector or CSN6. The cell lysates were immunoprecipitated with anti-Snail1 and immunoblotted with anti-ubiquitin antibody. 


\section{Discussion}

Accumulating evidence has suggested that CSN plays an important role in the regulation of multiple cancers and could be an effective target for therapeutic intervention. In addition, CSN is involved in regulating cullin-based E3 ligases and could potentially be altered in tumors [32, 33]. Previous studies have shown that CSN3 and CSN5 could accelerate the cancer cell proliferation and growth $[34,35]$. CSN6, as a critical subunit of CSN, is upregulated in numerous malignances [20,36, 37]. It is identified that CSN6 is implicated in modulating the biological functions of cancer progression, including the DNA repair, cell cycle and tumorigenesis [16, 38]. Given these vital roles, a better perceptive of the biological functions of CSN6 signaling regulation will provide potential approaches for inhibiting CSN6-induced cancerogenesis. Although few studies documented the roles of CSN6 in tumorigenesis, the mechanism roles of CSN6 in breast cancer tumorigenesis remain to be determined. Our study reveals that CSN6 promotes the migration and metastasis of breast cancer cells, indicating that CSN6 functions as an oncogene in breast cancer cells.

In the last several years, abnormal expression of CSN6 has been revealed to be associated with the clinicopathological characteristics of tumors and the prognosis of several tumor patients. The present study has suggested that CSN6 is not only highly expressed in tumors, but also plays an important role in the biological behavior of tumor cells. It is reported that CSN6 could stabilize the E3 ubiquitin ligase COP1 in human colorectal cancer cell line HCT116 and human embryonic kidney cell line HEK-293T, thereby accelerating the cyclin-dependent protein kinase inhibitor p27 that involved in the G1 phase of cell cycle to regulate cell proliferation [39]. In addition, overexpression of CSN6 could also reduce the ubiquitination of the proto-oncoprotein c-Jun mediated by the E3 ubiquitin ligase MEKK1 and promote cell proliferation in the human embryonic kidney cell lines [40]. In glioma cells, CSN6 stabilizes epidermal growth factor receptor (EGFR) by enhancing the self-ubiquitination of E3 ubiquitin ligase CHIP, thereby promoting glioma cells proliferation, migration and invasion [17]. Meanwhile, CSN6 could bind to the E6 oncoprotein of human papillomavirus (HPV) and inhibit the polyubiquitination of E6 to reduce its degradation, thereby regulating the activity of p53 in cell proliferation and apoptosis to affect the development of cervical cancer [41]. These studies indicated that CSN6 was carcinogenic in the occurrence and development of cancer. In the current study, we

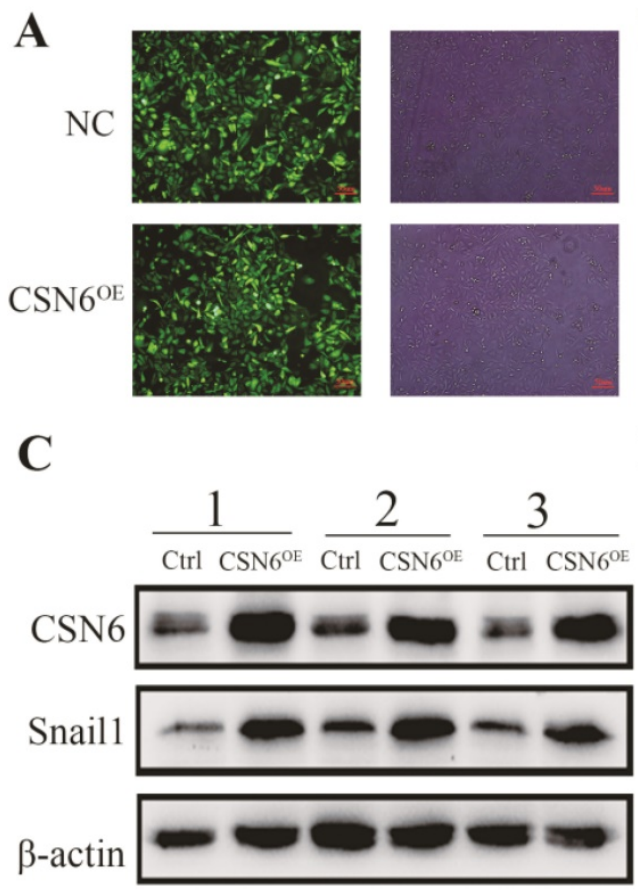

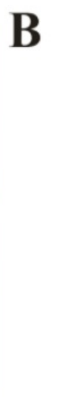

D

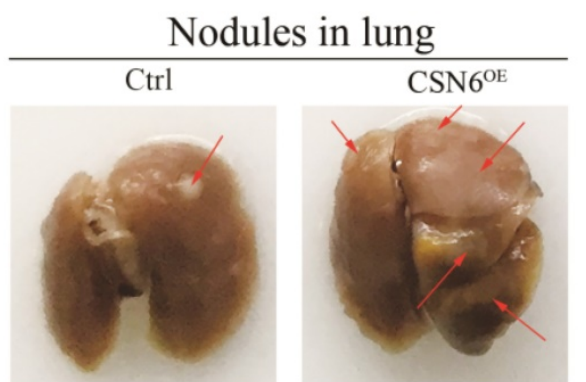

Normal lung tissue Metastatic lung tissue
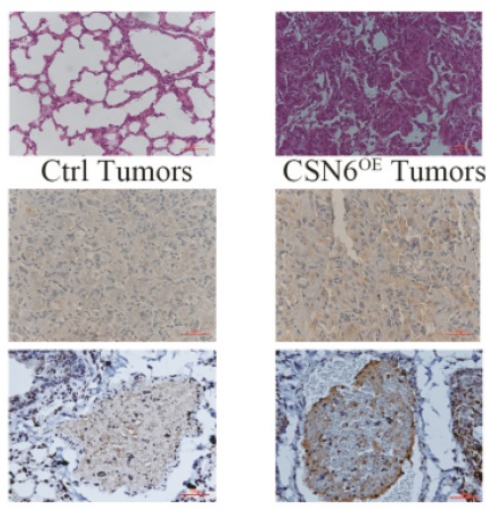

$\mathrm{HE}$

IHC(CSN6)

$\operatorname{IHC}($ Snaill)

Figure 4. CSN6 promotes breast cancer cells metastasis in vivo. (A) The photograph of MDA-MB-231 cells stably transfected CSN6 under a fluorescence microscope. (B) Numbers of lung metastasis nodules from each mouse were counted. (C) Western blot detected the protein expressions of CSN6 and Snail1 in metastasis tumors. (D) H\&E staining of lung sections. Representative images of IHC for CSN6 and Snaill staining metastasis tumors (Original magnifications, $400 \times$ ). The data represent means \pm S.D. $* * * P<$ 0.001 . 
investigated the role of the CSN6 in breast cancer and found that the expression of CSN6 in breast cancer cell lines was higher than that of normal breast epithelial cell lines. We also collected 52 pairs of breast cancer tissues and corresponding para-cancerous tissues for immunohistochemistry analysis. The results revealed that the expression of CSN6 in cancer tissues was higher than that of para-cancerous tissues, and it was significantly related to TNM staging. These results suggest that CSN6 plays a critical role in the progress of breast cancer. Subsequently we investigated the biological function of CSN6 in breast cancer.

Although the combined application of surgical treatment, radiotherapy and chemotherapy has greatly improved the five-year survival rate of breast cancer patients, the cancer recurrence still plagues various patients. The recurrent breast cancer frequently has distant metastasis of tumors, making the patient lost the opportunity for surgery. EMT is an important biological process in which malignant tumor cells derived from epithelial cells and acquire the capabilities of migration and invasion [42, 43]. During EMT, the expression of epithelial marker gene E-cadherin decreases and the expression of interstitial marker genes increases, including Snail, N-cadherin and vimentin [42]. Snail, an important zinc finger transcription factor that drives the EMT process, includes three members Snail1 (Snail), Snail2 (Slug) and Snail3 (Smuc). They bind to the E-box DNA sequence through the carboxyl terminal zinc finger domain to inhibit the expression of epithelial marker genes [44]. Studies have demonstrated that the activity of Snail1 is enhanced in the vicinity of the promoter of the epithelial marker E-cadherin, thereby inhibiting the expression of E-cadherin and promoting the progress of EMT [45]. In addition to inhibiting the expression of epithelial marker genes, Snail1 could also activate gene expression of interstitial phenotypes, such as fibronectin, $\mathrm{N}$-cadherin, and matrix metalloproteinase family [46]. This study we investigated the roles of CSN6 in breast cancer cells by specifically interfering or overexpressing CSN6. CSN6 overexpression conferred on cells tumorigenic properties and contributed to migration of breast cancer cells in vitro and in vivo, CSN6 knockdown resulted in a reverse trend. Western Blot analysis showed that CSN6 could up-regulate the expression of Snaill protein in a dose-dependent manner, and CO-IP analysis showed that CSN6 could bind with Snail1. These results indicate that CSN6 affects the EMT process of cancer cells by up-regulating the expression of Snail1 protein and promotes cell migration.

Next, we investigated how CSN6 specifically regulates the protein level of Snail1. Through RT-PCR analysis we found that the mRNA level of Snail1 did not change significantly after CSN6 overexpression in breast cancer cell lines. Thus, we speculated that CSN6 induced up-regulation of Snail may be regulated at the post-transcriptional level. It is identified that the CSN plays an important role in protein degradation because it has a high degree of homology with the "lid" complex of the 265 proteasome in mammals [8]. Previous studies have shown that CSN6 play an important role in the regulation of proteasome activity depending on the $\mathrm{N}$-terminal domain of Mprl/Pad1 [10-12]. Our study showed that CSN6 prevented Snail1 degradation via proteasomal degradation. In many cases, modulation of protein levels largely relies on ubiquitinationmediated degradation by the 265 proteasome. Increasing evidence illustrates that the deubiquitination activity of CSN5 is critical for cancer progression [47]. Previous studies have shown that CSN5 led to the metastasis and EMT activation of cancer cells through decreasing ZEB1 ubiquitination [48]. We then validated whether ubiquitination is involved in CSN6-mediated Snail1 regulation. Ubiquitination assay was performed and revealed that CSN6 overexpression decreased the ubiquitination level of Snail1. The above results suggest that CSN6 promotes Snail1 stability via impeding ubiquitination of Snail1. On the basis of our data, it is possible that CSN6-mediated Snail1 stabilization may be a common feature of various types of cancer. Our discovery of CSN6 in stabilizing Snail1 updates the paradigm with evidence that CSN has a versatile role in modulating various types of E3 ligases. CSN6 probably functions to inhibit Snail1 ubiquitination and then switches the E3 ligase target from Snail1 itself to other proteins. Such a hypothesis is worth further investigation.

In summary, we draw noteworthy conclusions that CSN6 had a positive impact on Snail1 stability, and then contributing to the enhanced metastasis potential of breast cancer cells. Furthermore, CSN6 is associated with clinicopathologic parameters in breast cancer and it could act as valid prognostic factor of breast cancer. CSN6 induces EMT and enhances metastasis of breast cancer cells by reducing Snail1 ubiquitination. Therefore, CSN6 might be considered as a promising novel therapeutic target for breast cancer.

\section{Acknowledgements}

This work was supported by the National Natural Science Foundation of China (No. 81872080, 81572349), Jiangsu Provincial Medical Talent (ZDRCA2016055), the Science and Technology Department of Jiangsu Province (BK20181148), the 
Priority Academic Program Development of Jiangsu Higher Education Institutions (PAPD) and the 333 high-level talents of Jiangsu Province (BRA2019083).

\section{Competing Interests}

The authors have declared that no competing interest exists.

\section{References}

1. Veronesi U, Boyle P, Goldhirsch A, et al. Breast cancer. Lancet 2005;365:1727-41.

2. Wang $\mathrm{X}, \mathrm{Lu} \mathrm{H}$, Urvalek AM, et al. KLF8 promotes human breast cancer cell invasion and metastasis by transcriptional activation of MMP9. Oncogene 2011;30:1901-11.

3. Collaborative Group on Hormonal Factors in Breast C. Familial breast cancer: collaborative reanalysis of individual data from 52 epidemiological studies including 58,209 women with breast cancer and 101,986 women without the disease. Lancet 2001;358:1389-99.

4. Veronesi U, Paganelli G, Viale G, et al. A randomized comparison of sentinelnode biopsy with routine axillary dissection in breast cancer. The New England journal of medicine 2003;349:546-53.

5. Wei N, Chamovitz DA, Deng XW. Arabidopsis COP9 is a component of a novel signaling complex mediating light control of development. Cell 1994;78:117-24.

6. Wei N, Deng XW. The COP9 signalosome. Annual review of cell and developmental biology 2003;19:261-86.

7. Zhang SN, Pei DS, Zheng JN. The COP9 signalosome subunit 6 (CSN6): a potential oncogene. Cell division 2013;8:14.

8. Li L, Deng XW. The COP9 signalosome: an alternative lid for the $26 \mathrm{~S}$ proteasome? Trends in cell biology 2003;13:507-9.

9. Guo H, Jing L, Cheng Y, et al. Down-regulation of the cyclin-dependent kinase inhibitor p57 is mediated by Jab1/Csn5 in hepatocarcinogenesis. Hepatology 2016;63:898-913.

10. Gusmaroli G, Figueroa P, Serino G, et al. Role of the MPN subunits in COP9 signalosome assembly and activity, and their regulatory interaction with Arabidopsis Cullin3-based E3 ligases. The Plant cell 2007;19:564-81.

11. Peng Z, Serino G, Deng XW. Molecular characterization of subunit 6 of the COP9 signalosome and its role in multifaceted developmental processes in Arabidopsis. The Plant cell 2001;13:2393-407.

12. Lyapina S, Cope G, Shevchenko A, et al. Promotion of NEDD-CUL1 conjugate cleavage by COP9 signalosome. Science 2001;292:1382-5.

13. Maytal-Kivity V, Reis N, Hofmann K, et al. MPN+, a putative catalytic motif found in a subset of MPN domain proteins from eukaryotes and prokaryotes, is critical for Rpn11 function. BMC biochemistry 2002;3:28.

14. Chen B, Zhao R, Su CH, et al. CDK inhibitor p57 (Kip2) is negatively regulated by COP9 signalosome subunit 6 . Cell cycle 2012;11:4633-41.

15. Wang W, Tang M, Zhang L, et al. Clinical implications of CSN6 protein expression and correlation with mutant-type P53 protein in breast cancer. Japanese journal of clinical oncology 2013;43:1170-6.

16. Fang L, Lu W, Choi HH, et al. ERK2-Dependent Phosphorylation of CSN6 Is Critical in Colorectal Cancer Development. Cancer cell 2015;28:183-97.

17. Hou J, Deng Q, Zhou J, et al. CSN6 controls the proliferation and metastasis of glioblastoma by CHIP-mediated degradation of EGFR. Oncogene 2017;36:1134-44

18. Wen D, Liao T, Ma B, et al. Downregulation of CSN6 attenuates papillary thyroid carcinoma progression by reducing Wnt/beta-catenin signaling and sensitizes cancer cells to FH535 therapy. Cancer medicine 2018;7:285-96.

19. Mao Z, Sang MM, Chen C, et al. CSN6 Promotes the Migration and Invasion of Cervical Cancer Cells by Inhibiting Autophagic Degradation of Cathepsin L. International journal of biological sciences 2019;15:1310-24.

20. Chen J, Shin JH, Zhao R, et al. CSN6 drives carcinogenesis by positively regulating Myc stability. Nature communications 2014;5:5384.

21. Nieto MA, Huang RY, Jackson RA, et al. Emt: 2016. Cell 2016;166:21-45.

22. Nieto MA. The snail superfamily of zinc-finger transcription factors. Nature reviews Molecular cell biology 2002;3:155-66.

23. Parker BS, Argani P, Cook BP, et al. Alterations in vascular gene expression in invasive breast carcinoma. Cancer research 2004;64:7857-66.

24. Elloul S, Elstrand MB, Nesland JM, et al. Snail, Slug, and Smad-interacting protein 1 as novel parameters of disease aggressiveness in metastatic ovarian and breast carcinoma. Cancer 2005;103:1631-43

25. Blanco MJ, Moreno-Bueno G, Sarrio D, et al. Correlation of Snail expression with histological grade and lymph node status in breast carcinomas. Oncogene 2002;21:3241-6.

26. Cheng $\mathrm{CW}, \mathrm{Wu} \mathrm{PE}, \mathrm{Yu}$ JC, et al. Mechanisms of inactivation of E-cadherin in breast carcinoma: modification of the two-hit hypothesis of tumor suppressor gene. Oncogene 2001;20:3814-23.

27. Zhou BP, Deng J, Xia W, et al. Dual regulation of Snail by GSK-3beta-mediated phosphorylation in control of epithelial-mesenchymal transition. Nature cell biology 2004;6:931-40.
28. Imani S, Hosseinifard H, Cheng J, et al. Prognostic Value of EMT-inducing Transcription Factors (EMT-TFs) in Metastatic Breast Cancer: A Systematic Review and Meta-analysis. Scientific reports 2016;6:28587.

29. Kajita M, McClinic KN, Wade PA. Aberrant expression of the transcription factors snail and slug alters the response to genotoxic stress. Molecular and cellular biology 2004;24:7559-66.

30. Mani SA, Guo W, Liao MJ, et al. The epithelial-mesenchymal transition generates cells with properties of stem cells. Cell 2008;133:704-15.

31. Wu Y, Deng J, Rychahou PG, et al. Stabilization of snail by NF-kappaB is required for inflammation-induced cell migration and invasion. Cancer cell 2009:15:416-28

32. Richardson KS, Zundel W. The emerging role of the COP9 signalosome in cancer. Molecular cancer research : MCR 2005;3:645-53.

33. Wang $\mathrm{L}, \mathrm{Du} \mathrm{WQ}, \mathrm{Xie} \mathrm{M}$, et al. Jab1 promotes gastric cancer tumorigenesis via non-ubiquitin proteasomal degradation of p14ARF. Gastric cancer: official journal of the International Gastric Cancer Association and the Japanese Gastric Cancer Association. 2020.

34. Yu YS, Tang ZH, Pan QC, et al. Inhibition of Csn3 expression induces growth arrest and apoptosis of hepatocellular carcinoma cells. Cancer chemotherapy and pharmacology 2012;69:1173-80.

35. Wang L, Zheng JN, Pei DS. The emerging roles of Jab1/CSN5 in cancer. Medical oncology 2016;33:90.

36. Forozan F, Mahlamaki EH, Monni O, et al. Comparative genomic hybridization analysis of 38 breast cancer cell lines: a basis for interpreting complementary DNA microarray data. Cancer research 2000;60:4519-25.

37. Du W, Liu Z, Zhu W, et al. CSN6 promotes tumorigenesis of gastric cancer by ubiquitin-independent proteasomal degradation of p16(INK4a). Cancer biology \& medicine 2019;16:514-29.

38. Lee MH, Zhao R, Phan L, et al. Roles of COP9 signalosome in cancer. Cell cycle 2011;10:3057-66.

39. Choi HH, Guma S, Fang L, et al. Regulating the stability and localization of CDK inhibitor p27(Kip1) via CSN6-COP1 axis. Cell cycle 2015;14:2265-73.

40. Shin J, Phan L, Chen J, et al. CSN6 positively regulates c-Jun in a MEKK1dependent manner. Cell cycle 2015;14:3079-87.

41. Gao S, Fang L, Phan LM, et al. COP9 signalosome subunit 6 (CSN6) regulates E6AP/UBE3A in cervical cancer. Oncotarget 2015;6:28026-41.

42. Kalluri R, Weinberg RA. The basics of epithelial-mesenchymal transition. The Journal of clinical investigation 2009;119:1420-8.

43. Kalluri R. EMT: when epithelial cells decide to become mesenchymal-like cells. The Journal of clinical investigation 2009;119:1417-9.

44. Hao Y, Baker D, Ten Dijke P. TGF-beta-Mediated Epithelial-Mesenchymal Transition and Cancer Metastasis. International journal of molecular sciences. 2019;20.

45. Cano A, Perez-Moreno MA, Rodrigo I, et al. The transcription factor snail controls epithelial-mesenchymal transitions by repressing E-cadherin expression. Nature cell biology 2000;2:76-83

46. Lamouille S, Xu J, Derynck R. Molecular mechanisms of epithelialmesenchymal transition. Nature reviews Molecular cell biology 2014:15:178-96

47. Liu Y, Shah SV, Xiang X, et al. COP9-associated CSN5 regulates exosomal protein deubiquitination and sorting. The American journal of pathology 2009;174:1415-25.

48. Zhang S, Hong Z, Chai Y, et al. CSN5 promotes renal cell carcinoma metastasis and EMT by inhibiting ZEB1 degradation. Biochemical and biophysical research communications 2017;488:101-8. 